Ann. Zootech., I970, 19 (4), 46I-464.

NOTE:

\title{
UTILISATION ENERGÉTIQUE DE L'ORGE, DU BLÉ, DU MAÏS ET DU SORGHO PAR L'AGNEAU EN GROISSANCE
}

\author{
M. VERMOREL, P. THIVEND * et M. THÉRIEZ * \\ Station d'Études des Métabolismes, \\ * Station de Recherches sur l'Élevage des Ruminants, \\ Centre de Recherches de Clermont-Ferrand, \\ 63 - Saint-Genès-Champanelle \\ Institut national de la Recherche agronomique
}

\section{RÉSUMÉ}

L'étude de l'utilisation énergétique de 4 céréales, effectuée sur 86 agneaux en croissance par estimation de la composition corporelle, indique que la valeur énergétique nette du maïs est supérieure à celle de l'orge, du sorgho et du blé ; la valeur énergétique nette du blé est elle-même supérieure à celle de l'orge. Pour des agneaux ayant eu une vitesse de croissance modérée (de I63 à $\mathrm{r} 75 \mathrm{~g} / \mathrm{j}$ ) la protéinogenèse a été par rapport à la lipogenèse plus importante avec le maïs qu'avec les trois autres céréales.

\section{INTRODUCTION}

Les céréales représentent une part de plus en plus importante de la ration d'engraissement des ruminants. Or, en dehors des travaux de Kellner (1909) et de Nehring (1963), peu d'études ont été effectuées pour déterminer leur valeur énergétique. En particulier, les données concernant l'utilisation des produits terminaux de la digestion des céréales pour la croissance et pour l'engraissement des bovins et des ovins sont encore rares et partielles (ARMSTrong et BLAXTER, I96r). Pour ces différentes raisons, nous avons entrepris l'étude de l'utilisation digestive et énergétique de l'orge, du blé, du maïs et du sorgho par des agneaux en croissance recevant des régimes riches en céréales. Nous rapportons ici les premiers résultats relatifs à l'utilisation énergétique de ces différents régimes.

Nous avons comparé les quantités de protéines et de lipides fixés dans la carcasse par des agneaux ayant ingéré des quantités égales d'énergie métabolisable et d'azote digestible avec des régimes comportant une proportion élevée de céréales et la même quantité de foin. Cela nous a conduits à limiter les quantités d'aliments distribués et à introduire différentes proportions de tourteaux dans les rations.

Annales de Zootechnie. - I970. 


\section{MATÉRIEL ET MÉTHODES}

Quatre vingt-six agneaux Limousins ou Limousins $\times$ Romanov, non castrés, âgés de 9 à I 2 semaines, ont été répartis en 5 lots à un poids moyen de $19,8 \pm 2, \mathrm{I} \mathrm{kg}$; un lot témoin de $\mathrm{I}_{4}$ animaux a été abattu au début de l'expérience ; les quatre autres lots ont reçu un aliment concentré aggloméré comprenant de 65 , I à $70,8 \mathrm{p}$. Ioo de céréales, du tourteau de soja et un complément minéral, et un foin de luzerne normal de mauvaise qualité (I I,I p. Ioo de matières azotées totales). Les animaux ont été alimentés individuellement deux fois par jour ; ils ont été pesés pendant trois jours consécutifs à 7 heures, au début de la période expérimentale et avant l'abattage ; de plus, ils ont été pesés une fois chaque quinzaine.

Les animaux ont été abattus lorsqu'ils présentaient un état d'engraissement satisfaisant, à un poids moyen de $32,4 \pm \mathrm{I}, 2 \mathrm{~kg}$. Après ressuyage des carcasses, nous avons prélevé l'épaule gauche; nous en avons disséqué et pesé les différents tissus (muscles, tissus adipeux, os et déchets). Pour chaque lot, nous avons regroupé puis broyé les mêmes tissus et nous en avons prélevé trois échantillons pour en đéterminer les teneurs en matière sèche, en énergie brute et en azote. Nous avons estimé la composition des carcasses de deux façons en calculant:

$1^{0}$ les quantités des différents tissus à partir de la composition de 1'épaule disséquée, à l'aide des équations de régression établies par BoccArD;

$2^{\circ}$ les quantités d'énergie, de protéines et de lipides, en faisant l'hypothèse que la composition chimique des différents tissus de la carcasse est la même que celle des tissus correspondants de l'épaule.

L'estimation du poids de la carcasse à partir de la proportion des différents tissus dans l'épaule donne un écart systématique de plus $9,1 \mathrm{p}$. Ioo en début d'expérience et de moins 5,5 p. Ioo en fin d'expérience par rapport à la pesée de la carcasse froide (sans le gras périrénal et les rognons). L'écart de 9,I p. Ioo peut s'expliquer par le fait que les équations de régression que nous avons utilisées ont été établies avec des agneaux d'un poids moyen de $35 \mathrm{~kg}$; chez ces animaux, l'épaule représente $9,3 \mathrm{p}$. Ioo du poids de la carcasse ressuyée au lieu de Io, I p. Ioo chez les agneaux de $\mathbf{1 9}, 8 \mathrm{~kg}$ de poids vif du lot abattu au début de l'expérience. L'écart systématique observé avec les animaux abattus en fin d'expérience provient vraisemblablement de la différence de races (Limousine ou Limousine $\times$ Romanov au lieu de Ile-de-France, Charmoise et Berrichon du Cher utilisées par BoccarD). Nous avons donc corrigé l'estimation de la composition de la carcasse à partir des poids de carcasses ressuyées.

\section{RÉSULTATS ET DISCUSSION}

Le gain de poids vif des animaux a été modeste (de 163 à $175 \mathrm{~g} / \mathrm{j}$ ) parce que les quantités d'aliment distribué étaient limitées, le foin de mauvaise qualité, et parce que les animaux on $t$ souffert des fortes chaleurs de l'été pendant l'expérience. On n'a pas noté de différence entre les vitesses de croissance des animaux des 4 lots (tabl. I).

Les gains de poids des carcasses ont été identiques pour les animaux des lots orge et blé mais plus importants pour les animaux du lot sorgho et surtout pour ceux du lot maîs qui ont eu le rendement le plus élevé (tabl. I). Ces écarts peuvent s'expliquer par les différences de poids frais des contenus digestifs.

Les animaux des lots " orge " et "blé ", qui ont ingéré des quantités égales de matière sèche et d'énergie brute, ont formé les mêmes quantités de muscles et fixé des quantités voisines de protéines, ce qui indique que les deux céréales ont été utilisées de la même façon pour la protéinogenèse. Les animaux du lot "sorgho " ont formé un peu plus de muscles et de protéines que ceux des lots précédents mais ont ingéré 5 à 6 p. too de plus de matière sèche et d'énergie brute, ce qui ne permet pas d'attribuer au sorgho une action plus favorable sur la protéinogenèse que celle de l’orge ou du blé. 
Les animaux du lot maïs ont formé davantage de muscles (respectivement I5, I 7 et I 4 p. IOo) et fixé davantage de protéines que les animaux des lots orge, blé et sorgho. Cependant, ils n'ont pas consommé davantage d'énergie et de matière sèche que les agneaux des lots "orge " et "blé " et même 6 p. Ioo de moins que ceux du lot «sorgho ». De plus, les quantités de matières azotées digestibles ingérées ont été très voisines pour tous les lots. La supériorité du maïs traduit vraisemblablement une meilleure utilisation des produits terminaux de la digestion pour la protéinogenèse.

Les animaux du lot " maïs " ont fixé I6 et ro p. Ioo de plus d'énergie, I $_{4}$ et 9 p. Ioo de plus de tissus adipeux que ceux des lots " orge " et "blé ". Ces différences traduisent une valeur énergétique nette du maïs supérieure à celle du blé, elle-même plus élevée que celle de l'orge, puisque les consommations de matière sèche totale et de foin ont été comparables dans les trois lots et que la valeur énergétique nette du tourteau de soja est voisine de celle des céréales. Ces résultats sont en accord avec les données bibliographiques obtenues avec des animaux adultes.

TABLEAU I

\begin{tabular}{|c|c|c|c|c|c|c|c|c|c|c|c|c|c|c|c|}
\hline \multicolumn{3}{|c|}{$\begin{array}{l}\text { Composition du } \\
\text { régime }(\% \text { M.S.) }\end{array}$} & \multirow[b]{2}{*}{ 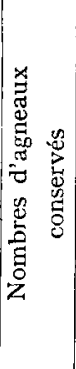 } & \multirow[b]{2}{*}{ 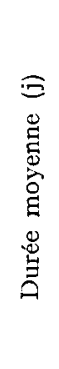 } & \multicolumn{3}{|c|}{ Quantités ingérées } & \multirow[b]{2}{*}{ 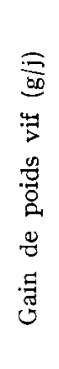 } & \multirow[b]{2}{*}{ 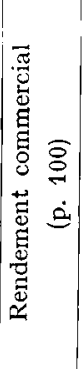 } & \multirow[b]{2}{*}{ 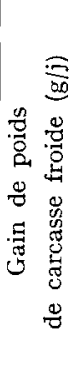 } & \multicolumn{5}{|c|}{$\begin{array}{l}\text { Quantités fixées } \\
\text { dans la carcasse }\end{array}$} \\
\hline $\begin{array}{l}\text { 号 } \\
\text { 恧 }\end{array}$ & 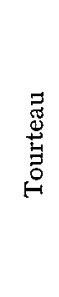 & : & & & 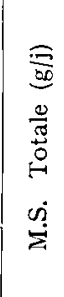 & 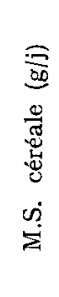 & 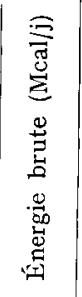 & & & & 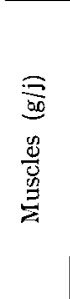 & 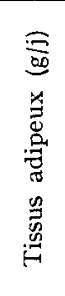 & 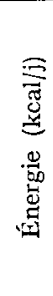 & 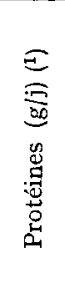 & 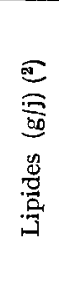 \\
\hline $\begin{array}{l}70,8 \\
\text { Orge }\end{array}$ & 7,3 & 18,6 & 17 & 70,4 & 906 & 641 & 3,93 & 175 & 48,2 & 98 & $\begin{array}{l}40,4 \\
54,5 *\end{array}$ & $\begin{array}{l}20,2 \\
23,4\end{array}$ & $\begin{array}{l}266 \\
322\end{array}$ & $\begin{array}{r}8,9 \\
13,0\end{array}$ & $\begin{array}{l}22,6 \\
26,1\end{array}$ \\
\hline $\begin{array}{l}68,8 \\
\text { Blé }\end{array}$ & 8,0 & $\mathbf{2 0 , 0}$ & 15 & 69,7 & 909 & 625 & 3,93 & 163 & 48,3 & 95 & $\begin{array}{l}40,8 \\
53,7\end{array}$ & $\begin{array}{l}21,5 \\
24,5\end{array}$ & $\begin{array}{l}288 \\
341\end{array}$ & $\begin{array}{r}8,7 \\
12,4\end{array}$ & $\begin{array}{l}25,1 \\
28,5\end{array}$ \\
\hline $\begin{array}{l}65, \overline{5} \\
\text { Maĩs }\end{array}$ & 12,1 & 19,1 & 15 & 67,9 & 898 & 588 & 3,97 & $17 / 4$ & 50,0 & 111 & $\begin{array}{l}46,5 \\
62,9\end{array}$ & $\begin{array}{l}22,9 \\
26,7\end{array}$ & $\begin{array}{l}307 \\
374\end{array}$ & $\begin{array}{l}11,4 \\
16,1\end{array}$ & $\begin{array}{l}25,5 \\
29,7\end{array}$ \\
\hline $\begin{array}{l}65,1 \\
\text { Sorgho }\end{array}$ & 12,5 & 19,1 & 14 & 68,6 & 948 & 617 & 4,17 & 163 & 50,0 & 104 & $\begin{array}{l}42,2 \\
55,1\end{array}$ & $\begin{array}{l}25,5 \\
28,4\end{array}$ & $\begin{array}{l}328 \\
380\end{array}$ & $\begin{array}{r}9,6 \\
13,4\end{array}$ & $\begin{array}{l}28,7 \\
32,0\end{array}$ \\
\hline
\end{tabular}

(1) $\mathrm{N} \times 6,25$.

${ }^{\left({ }^{2}\right)}$ Calculés par différence entre l'énergie fixée totale et l'énergie des protéines fixées.

* Les chiffres en italiques correspondent aux valeurs corrigées (cf. texte).

La valeur énergétique nette du sorgho est inférieure à celle du maïs du fait que les animaux du lot "sorgho " ont ingéré $6 \mathrm{p}$. roo de plus de matière sèche et fixé seulement $1,5 \mathrm{p}$. roo de plus d'énergie. On ne peut cependant pas classer le sorgho par rapport à l'orge et au blé en raison des différences dans les quantités de matière sèche ingérée et d'énergie fixée.

En comparant les quantités de protéines et de lipides fixés par jour, on constate que le blé et le sorgho favorisent la lipogenèse par rapport à l'orge et surtout au maïs. En effet, si le maïs a augmenté les quantités de tissus adipeux et de lipides fixés, par rapport à l'orge et au blé, il a accru encore davantage les quantités de muscles et de protéines fixées le rapport protéines fixées/ 
lipides fixés étant de 0,50 pour l'orge, 0,44 pour le blé, 0,54 pour le maïs et o, $4^{2}$ pour le sorgho. Nos résultats ont été obtenus avec des agneaux dont la vitesse de croissance a été modérée et doivent être vérifiés avec des animaux réalisant des gains de poids journaliers plus importants.

Reçu pour publication en septembre 1970.

\title{
SUMMARY
}

\author{
UTILIZATION OF THE ENERGY OF BARLEY, WHEAT, \\ MAIZE AND SORGHUM BY THE GROWING LAMB.
}

The utilization of the energy of 4 cereals was studied in 86 growing lambs by estimating the body composition and the results showed that the net energy value of maize is higher than that of barley, sorghum or wheat. The net energy value of wheat is superior to that of barley. In the lambs having a moderate growth rate (I63-I75 g/day), the proteinogenesis, compared with the lipogenesis, was more important with maize than with the three other cereals.

\section{RÉFÉRENCES BIBLIOGRAPHIQUES}

Armstrong D. G., Blaxter K. L., 196r. The utilization of the energy of carbohydrates by ruminant in 2th Symposium on energy metabolism Wageningen, p. 178).

BOCCARD R. (Communication personnelle.)

Kellner O., 1909. Die Ernährung der landteirschaftlichen Nutztiere. Ed. Verlag Paul Parey, Berlin.

Nehring K., Schiemann R., Hoffmann L., Jentsch W., ig63. Die energetische Verwertung der Futterstoffe. Arch. Tierernähr., 13, I19-213. 